\title{
Fecal calprotectin in inflammatory bowel disease
}

This article was published in the following Dove Press journal:

Clinical and Experimental Gastroenterology

28 January 2016

Number of times this article has been viewed

\section{Natalie E Walsham' \\ Roy A Sherwood ${ }^{2}$}

'Department of Clinical Biochemistry, University Hospital Lewisham, Lewisham, ${ }^{2}$ Department of Clinical Biochemistry, Viapath at King's College Hospital NHS Foundation Trust, London, UK
Correspondence: Roy A Sherwood Department of Clinical Biochemistry, King's College Hospital NHS Foundation Trust, Denmark Hill, London SE5 9RS, UK Email roy.sherwood@nhs.net
Abstract: Inflammatory bowel disease (IBD) and irritable bowel syndrome share many symptoms. While irritable bowel syndrome is a functional bowel disorder for which no specific treatment is available, the range of effective therapies for IBD is evolving rapidly. Accurate diagnosis of IBD is therefore essential. Clinical assessment, together with various imaging modalities and endoscopy, has been the mainstay of diagnosis for many years. Fecal biomarkers of gastrointestinal inflammation have appeared in the past decade, of which calprotectin, a neutrophil cytosolic protein, has been studied the most. Crohn's disease and ulcerative colitis are chronic remitting and relapsing diseases, and objective assessment of disease activity and response to treatment are important. This review focuses on the use of fecal calprotectin measurements in the diagnosis and monitoring of patients with IBD.

Keywords: calprotectin, Crohn's disease, ulcerative colitis, inflammatory bowel disease, inflammation

\section{Introduction}

Lower bowel symptoms, including chronic abdominal pain or discomfort with diarrhea or constipation, are common presenting features in both primary and secondary care settings. These symptoms may be caused by a number of different conditions, including inflammatory bowel disease (IBD), of which ulcerative colitis (UC) and Crohn's disease (CD) are the most common, and irritable bowel syndrome (IBS). IBS is a functional bowel disorder for which there is no identifiable cause or distinctive pathology, and treatment is symptomatic. Although it may be sufficiently troublesome to interfere with normal daily activities, it is seldom associated with serious morbidity. The prevalence of IBS has been estimated to be in between $10 \%$ and $20 \%$ of the adult population, but may be even higher than this as it is thought that many people with IBS symptoms do not seek medical assistance. ${ }^{1}$ The most common age of presentation is between the ages of 20 years and 30 years, although it is increasingly being seen at older ages, and is twice as common in women than in men.

$\mathrm{UC}$ and CD are the two most common forms of IBD. The prevalence of UC is approximately 100-200 per 100,000 people, and the prevalence of CD is approximately $50-100$ per 100,000 people,${ }^{1}$ with no significant sex difference. IBD is more common in Caucasians compared to those of Asian or Afro-Caribbean origin. The typical age of presentation is between 15 years and 30 years, but in up to $20 \%$, it presents during childhood. Both $\mathrm{UC}$ and $\mathrm{CD}$ are remitting and relapsing conditions with a variable course of progression. The prognosis of patients with $\mathrm{CD}$ is worse than that for $\mathrm{UC}$, although it is estimated that $10 \%$ of patients with $\mathrm{UC}$ will require a colectomy within 
10 years of diagnosis. Because of the significance of making a diagnosis of IBD versus IBS, there has been significant interest in developing biomarkers that can aid diagnosis.

The conventional diagnostic pathway includes initial blood tests, including a full blood count to exclude anemia, markers of inflammation such as the erythrocyte sedimentation rate (ESR) and C-reactive protein (CRP), plus serological testing for celiac disease (typically tissue transglutaminase antibodies). ESR and CRP, while specific for inflammation, cannot localize it to the gut. These initial blood tests are used to assist in deciding which patients should then proceed to imaging studies and/or endoscopy.

\section{Calprotectin}

Calprotectin is a small calcium-binding protein and is a member of the S100 family of zinc-binding proteins, being a heterodimer of S100A8/A9. It was first discovered in 1980 and was found to contribute $\sim 60 \%$ of the protein content of the cytosol in neutrophils. ${ }^{2}$ It has also been detected in monocytes and macrophages, albeit at lower concentrations than in neutrophils and may have anti-microbial properties. ${ }^{3}$ In the presence of active intestinal inflammation, polymorphonuclear neutrophils migrate to the intestinal mucosa from the circulation. Any disturbance to the mucosal architecture due to the inflammatory process, results in leakage of neutrophils, and hence, calprotectin, into the lumen and its subsequent excretion in feces. ${ }^{4}$ The concentration of calprotectin in feces has been shown to correlate well with the "gold standard". ${ }^{111}$ Indium-labeled leukocyte test and to the severity of the intestinal inflammation. ${ }^{5}$ Calprotectin appears to be distributed homogeneously in feces and is stable for up to 7 days at room temperature. ${ }^{6}$ In a recent study of 18 patients with active UC in which a total of 287 stool samples were collected, however, there was no change in calprotectin concentrations at 3 days at room temperature, but a mean decrease of $28 \%$ after 7 days. ${ }^{7}$ However, there may be considerable day-to-day variation in fecal calprotectin concentrations among individuals, although studies have been contradictory. In a small study of 14 individuals with normal findings on colonoscopy providing eight stool samples each, a within-subject coefficient of variation (CV) of $58 \%$ was observed. ${ }^{8}$ Two studies of patients with active UC have produced similar results. One of the studies, in which 56 stool samples were collected from 18 subjects, found a median $\mathrm{CV}$ for calprotectin of $40 \%$, and the other with 18 patients and 287 samples, a median CV of $52 \%{ }^{7,9}$ However, in a larger study of 143 consecutive patients with $\mathrm{CD}$ in remission, only $16 \%$ of patients had a significant within-individual variability for calprotectin concentrations over three samples. ${ }^{10}$ Fecal calprotectin concentrations may vary with age. Newborn infants have higher fecal calprotectin concentrations that decline to adult values by the age of 5 years. ${ }^{11}$

\section{Methods for the measurement of fecal calprotectin}

The methods for the measurement of fecal calprotectin are all based on immunochemical techniques utilizing either polyclonal or monoclonal antibodies targeted at various epitopes on the dimeric calprotectin molecule. They can be divided into those that produce a quantitative result and those that produce a positive or negative result, that is, qualitative result. The latter are primarily designed for use in the point-of-care setting, and the result can either be read visually or with a metering device. The initial methods were all in-house enzyme-linked immunosorbent assay (ELISA) techniques, but commercial assays are now widely available and some are listed in Table 1 . The commercially available ELISAs are all very similar and can be carried out manually or semiautomated using various ELISA platforms. A disadvantage of the ELISA format is that they are a form of batch analysis with a typical batch being 35-40 samples. This has the effect of restricting the turnaround time for calprotectin measurements. A number of manufacturers have launched random access immunochemical methods based on fluorescent, chemiluminescent, or immunoturbidimetric detection in recent years. The analytical performance of all the quantitative assays is similar, but the random access methods permit greater flexibility in batch sizes and hence, turnaround times. A comparison of six fecal calprotectin methods has been published recently ${ }^{12}$

Table I Some commercially available assays for fecal calprotectin

\begin{tabular}{|c|c|}
\hline Manufacturer & Test \\
\hline \multirow[t]{4}{*}{ Bühlmann } & EK-CAL ELISA - quantitative (two versions) \\
\hline & Quantum Blue - rapid quantitative \\
\hline & immunochromatography (two versions) \\
\hline & FCAL Turbo - quantitative immunoturbidimetric \\
\hline Calpro & Calpro ELISA - quantitative (two versions) \\
\hline \multirow[t]{2}{*}{ Eurospital } & Calprest ELISA - quantitative \\
\hline & Calfast - rapid quantitative immunochromatography \\
\hline Immundiagnostik & ELISA - quantitative \\
\hline \multirow[t]{2}{*}{ Phadia } & Immunocap Elia Calprotectin - quantitative \\
\hline & fluorescence enzyme immunoassay \\
\hline Preventis & Immunochromatographic semiquantitative test \\
\hline Diasorin & Liaison $\mathrm{XL}$ - quantitative chemiluminescent \\
\hline & immunoassay \\
\hline Biotec & Certest - semiquantitative immunochromatography \\
\hline
\end{tabular}

Abbreviation: ELISA, enzyme-linked immunosorbent assay. 
and the performance of point-of-care calprotectin kits has also been the subject of a review. ${ }^{13}$

All the methods for fecal calprotectin require some form of sample pretreatment to extract the calprotectin from the stool samples into a buffer for analysis. The "gold standard" method for extraction is weighing a set amount of feces into the buffer. This is obviously a labor-intensive technique, and a number of extraction devices have become commercially available. These have been reported to have variable recoveries depending on the consistency of the stool with lower recoveries from watery stool samples. ${ }^{14}$

\section{Differentiation of IBS from IBD}

Patients with IBS or IBD often have symptoms in common, including abdominal pain, bloating, and diarrhea. Initial studies of fecal calprotectin focused on its ability to distinguish between these two conditions in patients referred to gastroenterology departments from primary care. In a study of 602 consecutive patients attending a gastroenterology clinic at King's College Hospital (London, UK), patients had fecal calprotectin measured and were assessed for IBS using the ROME questionnaire. ${ }^{15}$ Fecal calprotectin had $89 \%$ sensitivity and $79 \%$ specificity for the detection of organic bowel disease. The sensitivity of a positive ROME questionnaire for IBS was $85 \%$ at a specificity of $71 \%$. Combining the two, however, had a predictive value for IBS approaching $100 \%$.

In the past 10 years, there have been many studies of the value of fecal calprotectin in distinguishing IBD from IBS, and these have been summarized in several recent meta-analyses. Gisbert and $\mathrm{McNicholl}{ }^{16}$ combined data from 2,475 patients and obtained a mean sensitivity of $83 \%$ and specificity of $84 \%$ for calprotectin to distinguish organic and nonorganic disease. In this meta-analysis, studies were included that did not report histology from the right colon and/or terminal ileum, which may have resulted in microscopic inflammation being missed, potentially resulting in an underestimation of sensitivity and specificity. The diagnostic accuracy was higher for CD (sensitivity, 83\%; specificity, 85\%) compared to UC (sensitivity $72 \%$, specificity $74 \%$ ). von Roon et $\mathrm{al}^{17}$ analyzed data from 30 studies with a total of 5,983 patients and found a sensitivity of $95 \%$ and a specificity of $91 \%$. The earlier meta-analyses only included studies carried out in adults. Van Rheenen et $\mathrm{al}^{18}$ included both adults and children with suspected IBD (670 adults, 371 children). Diagnosis was, however, only confirmed by endoscopy and histology in $32 \%$ of adults and $61 \%$ of children. Pooled sensitivity and specificity for fecal calprotectin were $93 \%$ and $96 \%$, respectively.
A recent meta-analysis of fecal calprotectin in pediatric studies (nine studies including 853 patients) reported a sensitivity of $97 \%$ at a specificity of $70 \%$. Using a cutoff of $50 \mu \mathrm{g} / \mathrm{g}$, $17 \%$ false positive and $2 \%$ false negative results would have occurred. ${ }^{19}$ Incorporating calprotectin in the consideration of the need for endoscopy would have allowed a $67 \%$ reduction in endoscopies at the expense of $6 \%$ of cases having a delayed diagnosis of IBD due to a false negative result. The specificity was significantly lower in children compared to adults for the same sensitivity ( $76 \%$ in children vs $96 \%$ in adults). A subsequent meta-analysis that only included pediatric studies supported this difference in children. ${ }^{20} \mathrm{~A}$ total of 715 patients were included (394 with IBD and 321 without) and the resulting sensitivity and specificity were $98 \%$ and $68 \%$, respectively. A retrospective analysis of 298 patients undergoing colonoscopy at a center in Switzerland found that fecal calprotectin improved the diagnostic yield compared to just using the European Panel on the Appropriateness of Gastrointestinal Endoscopy (EPAGE) criteria. ${ }^{21}$

These studies were based on patients attending secondary or tertiary care clinics and may be subject to bias due to preselection of patients in primary care prior to referral. Two reasonably large studies in primary care settings have suggested that both sensitivity and specificity are lower when compared to those achieved in secondary and tertiary care. In a retrospective analysis of data from 946 patients from 48 primary care practices, a sensitivity of $82 \%$ and a specificity of $77 \%$ were obtained. ${ }^{22} \mathrm{~A}$ further study looking at pointof-care testing combined measurements of fecal calprotectin and fecal occult blood (using an immunochemical test) and found a sensitivity of $79 \%$ but a specificity of only $49 \% .^{13}$

\section{Assessment of disease activity and response to treatment Assessment of disease activity}

Calprotectin has been shown to differentiate between active and inactive IBD. ${ }^{23} \mathrm{~A}$ number of studies have related fecal calprotectin to endoscopically assessed disease activity. In a study in patients with IBD fecal calprotectin correlated well with endoscopic disease activity measured using the Rachmilewitz index $(r=0.834) .{ }^{24} \mathrm{~A}$ similar study in UC but using the modified Baron index also found that fecal calprotectin correlated better with endoscopic disease activity than did CRP ( $r=0.821$ vs 0.556 ) ${ }^{25}$ In CD, fecal calprotectin has been related to the Crohn's Disease Index of Severity (CDIS) $(r=0.729) .{ }^{26} \mathrm{~A}$ further study that compared fecal calprotectin with CDIS demonstrated a very high area under the receiver operating characteristic curve of $0.935,{ }^{27}$ with 
an optimal cutoff of $274 \mu \mathrm{g} / \mathrm{g}$. Fecal calprotectin has now been incorporated into guidelines for monitoring IBD disease activity in Switzerland. ${ }^{28}$

\section{Assessing response to treatment}

The aim of treatment in IBD is to secure and maintain remission, with the ultimate goal of achieving mucosal healing. ${ }^{29}$ Drug therapy includes steroids, aminosalicylates, thiopurines, methotrexate, immunosuppressants (eg, ciclosporin), and antibodies targeting tumor necrosis factor alpha (TNF-alpha) (eg, infliximab). Assessment of treatment efficacy has traditionally been based on subjective relief of symptoms, clinical scores such as Crohn's Disease Activity Index (CDAI) and Ulcerative Colitis Disease Activity Index (UCDAI), and changes in the ESR and/or the concentration of CRP. Roseth et al carried out colonoscopies in patients with IBD in clinical remission and found that in 38 of 45 patients with normal histology the fecal calprotectin concentration was within the reference range. ${ }^{30} \mathrm{~A}$ subsequent study in $\mathrm{CD}$ patients found that a calprotectin $<250 \mu \mathrm{g} / \mathrm{g}$ identified mucosal healing with $94 \%$ sensitivity and $62 \%$ specificity. ${ }^{31}$ Fecal calprotectin has been reported to identify endoscopic disease activity more reliably than the CDAI. Rises in calprotectin results longitudinally may indicate relapse earlier than the CDAI. ${ }^{32}$

The response of patients with CD to anti-TNF-alpha therapy was investigated by Sipponen et al;3 patients had colonoscopy at initiation of therapy and at 12 weeks. The median fecal calprotectin fell from $1,173 \mu \mathrm{g} / \mathrm{g}$ to $130 \mu \mathrm{g} / \mathrm{g}$ and values were within the reference range in five patients with endoscopic remission. Similar results have been demonstrated in UC using infliximab where a fast and significant fall in calprotectin concentrations occurred (median at baseline $1,260 \mu \mathrm{g} / \mathrm{g}$ and at week $1,073 \mu \mathrm{g} / \mathrm{g}){ }^{34}$ In a further study of 60 IBD patients (34 CD, $26 \mathrm{UC}$ ) treated with either infliximab $(n=42)$ or adalimumab $(n=18)$, the fecal calprotectin concentration had fallen to $<100 \mu \mathrm{g} / \mathrm{g}$ in $52 \%$ of patients, of whom $84 \%$ remained in remission at 1 year compared to only $38 \%$ whose fecal calprotectin concentrations remained $>100 \mu \mathrm{g} / \mathrm{g} \cdot{ }^{35}$ In severe acute UC, calprotectin may be able to predict which patients will not respond to anti-TNF-alpha therapy and will require surgical intervention. ${ }^{36}$ A prospective multicenter study that included 49 patients reported that an increase in fecal calprotectin occurred 2-6 months prior to endoscopic relapse. ${ }^{36}$ Similarly, fecal calprotectin may be able to identify those patients who will require high-dose steroid therapy. ${ }^{37}$

The studies cited earlier were all carried out in adult subjects with IBD, but the findings have been corroborated in pediatrics. Two studies, one using corticosteroids and one infliximab, have shown that fecal calprotectin concentrations fall with clinical improvement and that values within the reference range predict sustained remission. ${ }^{38,39}$ It has been recommended that inflammatory markers, including fecal calprotectin, should be measured before initiating or changing IBD therapy. ${ }^{40}$

\section{Prognosis}

\section{Prediction of disease relapse}

A number of studies have reported that fecal calprotectin can predict the relapse of IBD in the following 12 months in patients in remission. Costa et al demonstrated a 14-fold greater risk of relapse in UC patients if the calprotectin was $>150 \mathrm{~g} / \mathrm{g}$, but this did not hold true for CD patients. ${ }^{42}$ In the initial study by Tibble et al, the relapse rate was found to be $85 \%$ for patients with UC and CD whose fecal calprotectin results were above the cutoff of $50 \mathrm{~g} / \mathrm{g}$ compared to $10-15 \%$ where the results were $<50 \mathrm{~g} / \mathrm{g}$. ${ }^{43}$ In a study of children with IBD, 39\% relapsed when the fecal calprotectin was above $100 \mathrm{~g} / \mathrm{g}$ compared to only $25 \%$ whose results were below the cutoff. ${ }^{44}$ Similar results were found in a large study (97 patients with UC and 65 with CD) using a cutoff of $130 \mathrm{~g} / \mathrm{g}$ where $20 \%$ of patients relapsed when the calprotectin was below the cutoff, while $43 \%$ (CD) and 59\% (UC) relapsed if the result was above the cutoff. ${ }^{45}$ In the study by Gisbert et al, the baseline calprotectin concentrations in patients with IBD who relapsed within 12 months was significantly higher $(239 \mathrm{~g} / \mathrm{g}$ versus $136 \mathrm{~g} / \mathrm{g} ; P<0.001)$, which corresponded with a relapse rate of $30 \%$ when the calprotectin was $>150 \mathrm{~g} / \mathrm{g}$ compared to $7 \%$ at lower concentrations. ${ }^{46}$ In two pediatric studies, the relapse rate was significantly lower in patients whose fecal calprotectin was below the chosen cutoff ( 257 and $400 \mathrm{~g} / \mathrm{g}$ ) compared to those above the cutoff. ${ }^{47,48}$ Kallel et al found an 18 -fold risk of relapse in CD patients within 12 months if the calprotectin concentration was $>340 \mathrm{~g} / \mathrm{g} .{ }^{49}$ Using a cutoff of $150 \mathrm{~g} / \mathrm{g}$ in 60 patients with IBD, $25 \%$ of patients below the cutoff relapsed, whilst $75 \%$ of those above the cutoff suffered a relapse. ${ }^{50}$ Molander et al found that calprotectin concentrations $>139 \mu \mathrm{g} / \mathrm{g}$ predicted relapse after 12 months, with a sensitivity of $72 \%$ and specificity of $80 \% .{ }^{35}$ A different approach was taken by De Vos et al, who have suggested that it is the change in calprotectin results in the first 3 months of therapy that better predicts relapse in that two results $>300 \mu \mathrm{g} / \mathrm{g}$ during a month interval of treatment predicted relapse with $61 \%$ sensitivity and 100\% specificity. ${ }^{44}$ Clearly, a variety of cutoffs have been proposed 
to detect relapse and this is partly dependent on whether the authors chose to maximize sensitivity or specificity. The lower the cutoff the greater is the sensitivity at the expense of specificity and vice versa.

\section{Postoperative IBD recurrence}

Surgical treatment of $\mathrm{CD}$ with resection of the terminal ileum or colectomy in UC may be necessary in up to $20 \%$ of patients during their lifetime. ${ }^{45}$ Current recommendations are that in patients with $\mathrm{CD}$ following ileocolonic resection, colonoscopy should be carried out 6-12 months after surgery. ${ }^{46}$ A noninvasive biomarker with good predictive power to identify those without recurrence would be desirable to minimize patient inconvenience and discomfort. Several studies have shown that fecal calprotectin concentrations remain high postsurgery in those subjects who subsequently developed recurrence compared to those in remission whose values often fell within the reference range. ${ }^{27,47-49}$ An Australasian study of 135 patients with CD found that a fecal calprotectin $<100 \mu \mathrm{g} / \mathrm{g}$ had a negative predictive value of $91 \%$, which if applied in this study would have meant nearly half $(47 \%)$ of the postoperative endoscopies could have been avoided. ${ }^{50} \mathrm{~A}$ similar study of 85 patients with $\mathrm{CD}$ that used the same cutoff of $100 \mu \mathrm{g} / \mathrm{g}$ found $30 \%$ of colonoscopies could have been avoided. ${ }^{51}$ The studies of fecal calprotectin in postoperative $\mathrm{CD}$ patients have been described in greater detail in a recent review. ${ }^{52}$

Patients who have had colectomy for UC have a significant risk of developing pouchitis postsurgery. There are only a few studies on the value of fecal calprotectin in detecting patients with pouchitis. In the study by Thomas et al, ${ }^{53}$ all subjects with endoscopic and histological evidence of pouchitis had raised fecal calprotectin concentrations, whereas only two of 15 without had an increase in calprotectin. Johnson et $\mathrm{al}^{54}$ found that not only was fecal calprotectin increased in patients with pouchitis, but that the result correlated with the Pouch Disease Activity Index. One study found similar results in children after colectomy. ${ }^{55}$

\section{Fecal calprotectin in other gastrointestinal tract disorders Gastrointestinal tract malignancy}

It is important to remember that while fecal calprotectin has good specificity for gastrointestinal (GI) tract inflammation, it is not specific to IBD being increased due to any cause of inflammation throughout most of the gut. In one of the earliest studies on fecal calprotectin, Tibble et $\mathrm{al}^{56}$ compared fecal calprotectin to conventional guiac or immunochemical occult blood testing (FOB) in 62 patients with colorectal carcinoma and found $90 \%$ of patients with carcinoma had positive calprotectin results compared to only $58 \%$ for FOB. The overall sensitivity and specificity of calprotectin for colorectal carcinoma were reported as $79 \%$ and $72 \%$, respectively. These results have, however, not been replicated in two major studies from Norway and Italy, where the sensitivity for detection of colorectal cancer was only $27 \%$ and $56 \%$, respectively. ${ }^{57,58}$ The meta-analysis of von Roon et $\mathrm{al}^{17}$ mentioned earlier included 5,983 patients in total, 297 of whom were subsequently found to have colorectal carcinoma. The sensitivity of a single fecal calprotectin measurement to detect cancer, having excluded those found to have IBD, was only $36 \%$ at a specificity of $71 \%$. This may be explainable in that not all cancers provoke both an inflammatory response and a disruption of the cellular architecture necessary to allow passage of neutrophils, with their associated calprotectin, into the lumen of the gut, producing a false negative result.

\section{Nonsteroidal anti-inflammatory drugs}

Chronic use of nonsteroidal anti-inflammatory drugs (NSAIDs) can result in GI tract side effects in many patients, which are often asymptomatic and can result in anemia. The presence of NSAID enteropathy was associated with raised fecal calprotectin in one of the earliest studies of the test. ${ }^{59}$ That NSAIDs were directly involved in causing low-grade inflammation was demonstrated in a study of 40 healthy volunteers taking diclofenac (150 mg/day) for 14 days. Compared to baseline values $75 \%$ had fecal calprotectin concentrations that had increased (median $11 \mu \mathrm{g} / \mathrm{g}$ vs $82 \mu \mathrm{g} / \mathrm{g}$ ). ${ }^{60}$

\section{Infectious gastroenteritis}

Most studies of fecal biomarkers of GI tract inflammation have focused on chronic rather than acute diarrhea. More than half the population in the developed world and nearly $100 \%$ of subjects in the developing world will have at least one bout of infectious diarrhea each year. This is usually self-limiting and does not require medical care, but more severe cases of bacterial infections are commonplace in the hospital environment, both community acquired and those infections acquired during hospitalization for some other cause. One large study in India included 2,383 consecutive patients presenting with acute diarrhea and found a sensitivity and specificity for fecal calprotectin for the identification of bacterial GI tract infection of $83 \%$ and $87 \%$, respectively. ${ }^{61}$ These findings have 
subsequently been confirmed in studies in both the adult and pediatric populations. ${ }^{62-64}$ Fecal calprotectin concentrations have been found to be higher in bacterial compared to viral infections and to correlate well with severity.

\section{Diverticular disease}

Patients with complex diverticular disease have histological evidence of mucosal inflammation. Two studies have shown that fecal calprotectin concentrations $>60 \mu \mathrm{g} / \mathrm{g}$ predict the acute complications of diverticular disease. ${ }^{65,66}$

\section{Other fecal markers}

Calprotectin has been the most studied fecal marker of GI tract inflammation, but in recent years, other markers have appeared that may prove to either be superior or provide complementary information. These include other proteins expressed in the cytoplasm of neutrophils, such as lactoferrin, S100A12, polymorphonuclear elastase, and M2-PK, which is an isoform of pyruvate kinase expressed by rapidly dividing cells. These have been reviewed recently. ${ }^{67,68}$

\section{Discussion}

The differential diagnosis of organic bowel disease and IBS can be challenging. The improvements that are occurring with time in cross-sectional imaging such as magnetic resonance imaging (MRI) and computed tomography (CT) have resulted in some cases being identified prior to endoscopy, which remains the "gold standard" as it permits the histological examination of biopsy samples. MRI and CT are both relatively expensive modalities, and CT has the disadvantage of exposure to ionizing radiation. While a single CT for diagnostic purposes does not result in a significant radiation exposure, the use of CT for monitoring disease activity can produce a substantial radiation burden with time, and MRI is the preferred modality for this purpose, although it is not always available or well tolerated by all patients.

Colonoscopy is an invasive procedure that typically requires sedation of the patient, causes discomfort, and is relatively expensive. A suggested algorithm for the use of fecal calprotectin in the diagnostic pathway for IBD/IBS is given in Figure 1. The choice of a cutoff for differentiating IBS

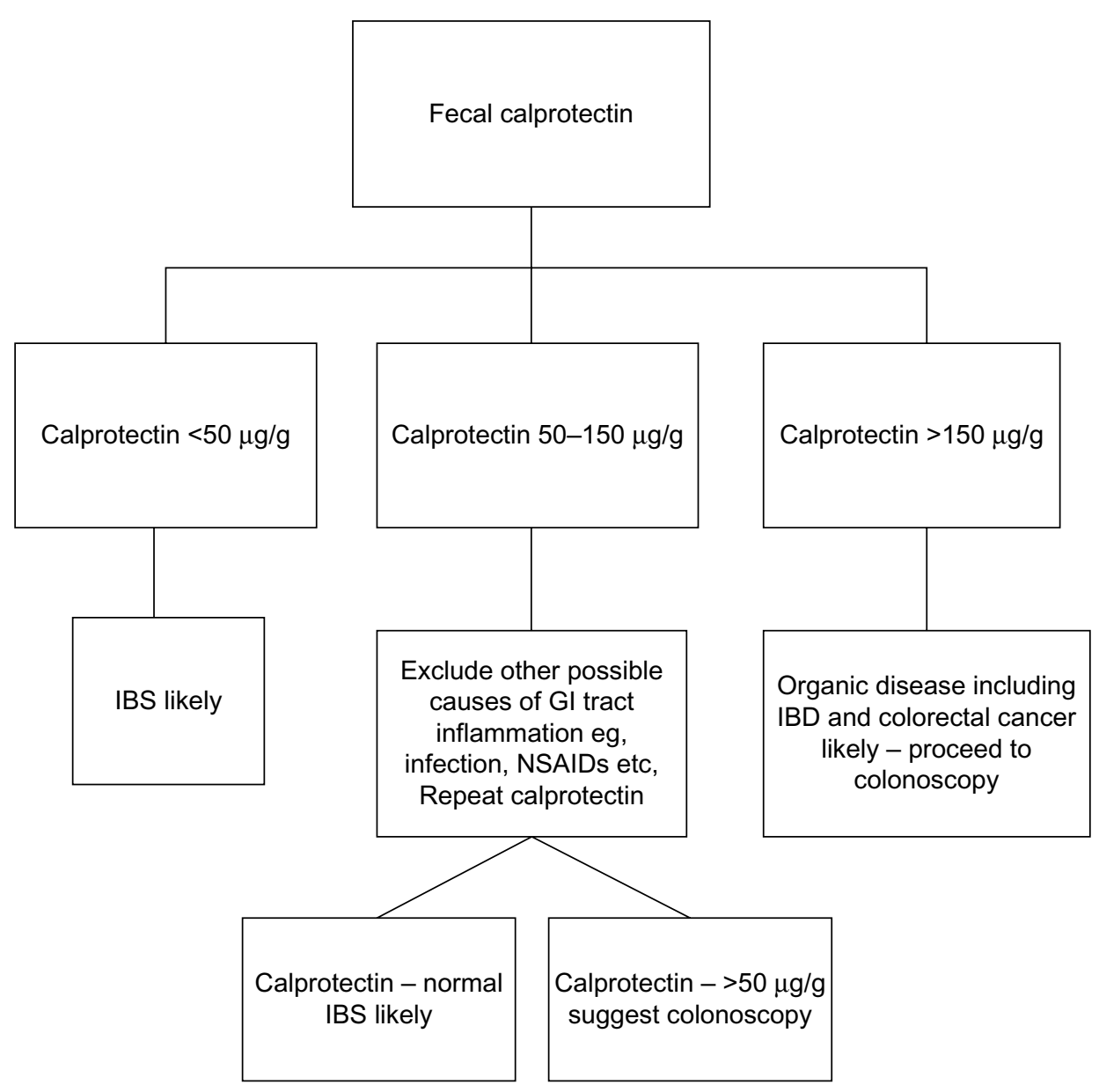

Figure I Suggested algorithm for the use of fecal calprotectin in the differentiation of IBS and IBD.

Abbreviations: IBS, irritable bowel syndrome; IBD, inflammatory bowel disease; GI, gastrointestinal; NSAIDs, nonsteroidal anti-inflammatory drugs. 
from IBD is an area of constant debate. The value of $50 \mu \mathrm{g} / \mathrm{g}$ is quoted by the majority of manufacturers of calprotectin kits and originates from the translation of the milligram per liter cutoff from the King's College Hospital initial study ${ }^{15}$ to microgram per gram. Increasing the cutoff will improve specificity at the expense of sensitivity. The choice of a cutoff will depend on whether sensitivity or specificity is considered to be the most important and needs to be made taking into consideration the clinical features of an individual patient. In a young patient, a cutoff of $150 \mu \mathrm{g} / \mathrm{g}$ may be applicable, whereas in an older patient in whom the risk of GI tract malignancy is higher, a lower cutoff could be better. As fecal calprotectin is increased in gastroenteritis associated with viral or bacterial infection a value between $50 \mu \mathrm{g} / \mathrm{g}$ and $150 \mu \mathrm{g} / \mathrm{g}$ should always be repeated 2-3 weeks later. Our experience is that up to $30 \%$ of such cases from primary care result in $<50 \mu \mathrm{g} / \mathrm{g}$ on repeat testing.

A few studies have investigated the cost-effectiveness of the use of fecal calprotectin in the diagnosis of IBD. Yang et al evaluated a financial model in 100 adults and 100 children screened with calprotectin prior to colonoscopy using $50 \mu \mathrm{g} / \mathrm{g}$ and $100 \mu \mathrm{g} / \mathrm{g}$ cutoffs. The use of calprotectin with a cutoff of $100 \mu \mathrm{g} / \mathrm{g}$ would have saved $\$ 417$ per patient in adults but would have delayed the diagnosis in $2.2 / 32$ adults and $\$ 300$ per patient in children but delayed the diagnosis in $4.8 / 61$ cases. A cutoff of $50 \mu \mathrm{g} / \mathrm{g}$ would have cost $\$ 55$ and $\$ 43$ more for adults and children, respectively, but yielded 2.4 and 6.1 additional accurate diagnoses of IBD per 100 subjects screened. ${ }^{69}$ The National Institute for Health and Clinical Excellence (NICE) review in 2013 concluded that the use of fecal calprotectin by primary care physicians prior to referral to a gastroenterology service was financially beneficial. ${ }^{1}$

\section{Conclusion}

The potential uses of the measurement of fecal calprotectin can be summarized as follows:

Diagnostic: Fecal calprotectin has adequate sensitivity and specificity to identify those patients most likely to have organic bowel disease, for example, IBD from functional disease, that is, IBS is therefore permitting effective management of colonoscopy resources.

Assessing disease activity and response to treatment: The aim of the treatment of IBD is to achieve mucosal healing, which can be identified noninvasively by normalization of fecal calprotectin.

Prognostic: A rising fecal calprotectin can predict an imminent clinical relapse of IBD allowing prompt initiation of treatment.
Research: A number of intestinal diseases have been found to have some degree of inflammation by using fecal calprotectin measurements even when this was not originally suspected.

\section{Disclosure}

The authors have no conflicts of interest with respect to the contents of this review.

\section{References}

1. National Institute for Health and Clinical Excellence. Faecal calprotectin diagnostic tests for inflammatory diseases of the bowel. NICE diagnostic guideline 11. London UK. 2013.

2. Fagerhol M, Dale I, Andersson T. A radioimmunoassay for a granulocyte protein as a marker in studies on the turnover of such cells. Bull Eur Physiopathol Respir. 1980;16(Suppl):273-282.

3. Hsu K, Champaiboon C, Guenther BD, et al. Anti-infective protective properties of S100 calgranulins. Antiinflamm Antiallergy Agents Med Chem. 2009;8:290-305.

4. Roseth A, Aadland E, Jahnsen J, Raknerud N. Assessment of disease activity in ulcerative colitis by fecal calprotectin, a novel granulocyte marker protein. Digestion. 1997;58:276-180.

5. Roseth A, Schmidt P, Fagerhol M. Correlation between faecal excretion of indium-111-labelled granulocytes and calprotectin, a granulocyte marker protein, in patients with inflammatory bowel disease. Scand J Gastroenterol. 1999;34:50-54.

6. Roseth A, Fagerhol M, Aadland E, Schjønsby H. Assessment of the neutrophil dominating protein in feces. A methodologic study. Scand J Gastroenterol. 1992;27:793-798.

7. Lasson A, Stotzer PO, Öhman L, Isaksson S, Sapnara M, Strid H. The intra-individual variability of faecal calprotectin: a prospective study in patients with active ulcerative colitis. J Crohns Colitis. 2015;9:26-32.

8. Husebye E, Tøn H, Johne B. Biological variability of fecal calprotectin in patients referred for colonoscopy without colonic inflammation or neoplasm. Am J Gastroenterol. 2001;96:2683-2687.

9. Calafat M, Cabré E, Manosa M, Lobatón T, Marín L, Domènech E. High within-day variability of fecal calprotectin levels in patients with active ulcerative colitis: What is the best timing for stool sampling? Inflamm Bowel Dis. 2015;21:1072-1076.

10. Naismith GD, Smith LA, Barry SJ, et al. A prospective single-centre evaluation of the intra-individual variability of faecal calprotectin in quiescent Crohn's disease. Aliment Pharmacol Ther. 2013;37:613-621.

11. Rugtveit J, Fagerhol MK. Age-dependent variations in fecal calprotectin concentrations in children. J Pediatr Gastroenterol Nutr. 2002;34: 323-324; author reply 324-325.

12. Labaere D, Smismans A, Van Olmen A, et al. Comparison of six different calprotectin assays for the assessment of inflammatory bowel disease. United European Gastroenterol J. 2014;2:30-37.

13. Kok L, Elias SG, Witteman BJ, et al. Diagnostic accuracy of point-ofcare fecal calprotectin and immunochemical occult blood tests for diagnosis of organic bowel disease in primary care: the Cost-Effectiveness of a Decision Rule for Abdominal Complaints in Primary Care (CEDAR) study. Clin Chem. 2012;58:989-998.

14. Whitehead SJ, French J, Brookes MJ, Ford C, Gama R. Between-assay variability of faecal calprotectin enzyme-linked immunosorbent assay kits. Ann Clin Biochem. 2013;50:53-61.

15. Tibble JA, Sigthorsson G, Foster R, Forgacs I, Bjarnason I. Use of surrogate markers of inflammation and Rome criteria to distinguish organic from nonorganic intestinal disease. Gastroenterol. 2002;123:450-460.

16. Gisbert JP, McNicholl AG. Questions and answers on the role of faecal calprotectin as a biological marker in inflammatory bowel disease. Dig Liver Dis. 2009;41(1): 56-66. 
17. Von Roon A, Karamountzos L, Purkayastha S, et al. Diagnostic precision of fecal calprotectin for inflammatory bowel disease and colorectal malignancy. Am J Gastroenterol. 2007;102:803-813.

18. Van Rheenen P, Van de Vijvier A, Fidler V. Faecal calprotectin for screening for patients with suspected inflammatory bowel disease: diagnostic meta-analysis. Brit Med J. 2010;341:c3369.

19. Degraeuwe PL, Beld MP, Ashorn M, et al. Faecal calprotectin in suspected paediatric inflammatory bowel disease. J Pediatr Gastroenterol Nutr. 2015;60:339-346.

20. Henderson P, Anderson N, Wilson D. The diagnostic accuracy of fecal calprotectin during the investigation of suspected pediatric inflammatory bowel disease: a systematic review and meta-analysis. $\mathrm{Am} \mathrm{J}$ Gastroenterol. 2014;109:637-645.

21. Burri E, Manz M, Schroeder P, et al. Diagnostic yield of endoscopy in patients with abdominal complaints: incremental value of faecal calprotectin on guidelines of appropriateness. BMC Gastroenterology. 2014;14:57.

22. Pavlidis P, Chedgy FJQ, Tibble JA. Diagnostic accuracy and clinical application of faecal calprotectin in adult patients presenting with gastrointestinal symptoms in primary care. Scand J Gastoenterol. 2013;48:1048-1054.

23. Langhorst J, Elsenbruch S, Koelzer J, Rueffer A, Michalsen A, Dobos G. Noninvasive markers in the assessment of intestinal inflammation in inflammatory bowel diseases: performance of fecal lactoferrin, calprotectin, and PMN-elastase, CRP and clinical indices. Am J Gastroenterol. 2008;103:162-169.

24. Schoepfer AM, Beglinger C, Strauman A, et al. Fecal calprotectin correlates more closely with the Simple Endoscopic Score for Crohn's disease (SES-CD) than CRP, leukocytes and the CDAI. Am J Gastroenterol. 2010;105:162-169.

25. Schoepfer AM, Beglinger C, Strauman A, et al. Fecal calprotectin more accurately reflects endoscopic activity of ulcerative colitis than the Lichtiger Index, C-reactive protein, platelets, hemoglobin and blood leukocytes. Inflamm Bowel Dis. 2013;19:332-341.

26. Sipponen T, Savilahti E, Kolho KL, Nuutinen H, Turunen U, Farkkila M. Crohn's disease activity assessed by fecal calprotectin and lactoferrin: correlation with Crohn's disease activity index and endoscopic findings. Inflamm Bowel Dis. 2008;14:40-46.

27. Lobatón T, López-García A, Rodríguez-Moranta F, Ruiz A, Rodríguez L, Guardiola J. A new rapid test for fecal calprotectin predicts endoscopic remission and postoperative recurrence in Crohn's disease. J Crohns Colitis. 2013;7:e641-e651.

28. Sauter B, Beglinger C, Girardin M, et al. Monitoring disease activity and progression in Crohn's disease. A Swiss perspective on the IBD Ahead 'Optimised Monitoring' recommendations. Digestion. 2014;89:299-309.

29. Schoepfer SM, Vavricka S, Zahnal-Straumann N, Straumann A, Beglinger C. Monitoring inflammatory bowel disease activity: clinical activity is judged to be more relevant than endoscopic severity or biomarkers. J Crohns Colitis. 2012;6:412-418.

30. Roseth A, Aadland E, Grzyb K. Normalization of fecal calprotectin: a predictor of mucosal healing in patients with inflammatory bowel disease. Scan J Gastroenterol. 2004;39:1017-1020.

31. D'Haens G, Fernante M, Vermiere S, et al. Fecal calprotectin is a surrogate marker for endoscopic lesions in inflammatory bowel disease. Inflamm Bowel Dis. 2012;18:2218-2224.

32. Burri E, Beglinger C, von Felten S, Lehmann FS. Fecal calprotectin and the clinical activity index are both useful to monitor medical treatment in patients with ulcerative colitis. Dig Dis Sci. 2015;60:485-491

33. Sipponen T, Savilahti E, Kärkkäinen P, et al. Fecal calprotectin, lactoferrin and endoscopic disease activity in monitoring anti-TNF-alpha therapy for Crohn's disease. Inflamm Bowel Dis. 2008;14:1392-1398.

34. De Vos M, Dewit O, D’Haens G, et al. Fast and sharp decline in calprotectin predicts remission by infliximab in anti-TNF naïve patients with ulcerative colitis. J Crohns Colitis. 2012;6:557-562.

35. Molander P, Af Björkestein C, Mustonen H, et al. Fecal calprotectin concentration predicts outcome in inflammatory bowel disease after induction therapy with TNF $\alpha$ blocking agents. Inflamm Bowel Dis. 2012;18:2011-2017.
36. Ho G, Lee H, Brydon G, Ting T, et al. Fecal calprotectin predicts the clinical course of acute severe ulcerative colitis. Am J Gastroenterol. 2009;104:673-678.

37. Molander P, Färkkilä M. Ristimäki A, et al. Does fecal calprotectin predict short-term relapse after stopping TNF $\alpha$-blocking agents in inflammatory bowel disease patients in deep remission? J Crohns Colitis. 2015;9:33-40.

38. Theede K, Kiszka-Kanowitz M, Mertz Nielsen A, Nordgaard-Lassen I. The correlation between fecal calprotectin, simple clinical colitis activity index and biochemical markers in ulcerative colitis during high-dose steroid treatment. Scand J Gastroenterol. 2014;49:418-423.

39. Kolho K, Raivio T, Lindahl H, Savilahti E. Fecal calprotectin remains high during glucocorticoids therapy in children with inflammatory bowel disease. Scand J Gastroenterol. 2006;41:720-725.

40. Kolho K, Sipponen T. The long-term outcome of anti-tumour necrosis factor- $\alpha$ therapy related to fecal calprotectin values during induction therapy in paediatric inflammatory bowel disease. Scan J Gastroenterol. 2014;49:434-446.

41. Colombel J, Sandborn W, Reinisch W, et al. Infliximab, azothioprine, or combination therapy for Crohn's disease. New Eng J Med. 2010;362:1383-1395.

42. Costa F, Mumolo MG, Ceccarelli L, et al. Calprotectin is a stronger predictive marker of relapse in ulcerative colitis than in Crohn's disease. Gut. 2005;54:364-368.

43. Tibble JA, Sigthorsson G, Bridger S, Fagerhol MK, Bjarnason I. Surrogate markers of intestinal inflammation are predictive of relapse in patients with inflammatory bowel disease. Gastroenterol. 2000;119:15-22.

44. Sipponen T, Kolho KL. Faecal calprotectin in children with clinically quiescent inflammatory bowel disease. Scand J Gastroenterol. 2010;45: 872-877.

45. D'Incà R, Dal Pont E, Di Leo V, et al. Can calprotectin predict relapse risk in inflammatory bowel disease. Am $J$ Gastroenterol. 2008;103:2007-1014

46. Gisbert JP, Bermejo F, Perez-Calle JL, et al. Fecal calprotectin and lactoferrin for the prediction of inflammatory bowel disease relapse. Inflamm Bowel Dis. 2009;15:1190-1198.

47. Diamanti A, Colistro F, Basso MS, et al. Clinical role of calprotectin assay in determing histological relapses in children affected by inflammatory bowel diseases. Inflamm Bowel Dis. 2008;14:1229-1235.

48. Walkiewicz D, Werlin SL, Fish D, Scanlon M, Hanaway P, Kugathasan S. Fecal calprotectin is useful in predicting disease relapse in pediatric inflammatory bowel disease. Inflamm Bowel Dis. 2008;14:669-673.

49. Kallel L, Ayadi I, Matri S, et al. Fecal calprotectin is a predictive marker of relapse in Crohn's disease involving the colon: a prospective study. Eur J Gastroenterol Hepatol. 2010;22:340-345.

50. Garcia-Sanchez V, Iglesias-Flores E, Gonzalez R, et al. Does fecal calprotectin predict relapse in patients with Crohn's disease and ulcerative colitis? J Crohns Colitis. 2010;4:144-152.

51. Vos MD, Louis EJ, Jahnsen J, et al. Consecutive fecal calprotectin measurements to predict relapse in patients with ulcerative colitis receiving infliximab maintenance therapy. Inlamm Bowel Dis. 2013;19:211-2117.

52. Ordás I, Eckmann L, Talamini M, Baumgart DC, Sandborn WJ. Ulcerative colitis. Lancet. 2012;380:1606-1619.

53. Van Assche G, Dignass A, Reinisch W, et al. The second European evidence-based concensus on the diagnosis and management of Crohn's disease: Special situations. J Crohns Colitis. 2010;4:63-101.

54. Lamb CA, Mohiuddin MK, Gicquel J, et al. Faecal calprotectin or lactoferrin can identify postoperative recurrence in Crohn's disease. Br J Surg. 2009;96:663-674.

55. Orlando A, Modesto I, Castiglione F, et al. The role of calprotectin in predicting endoscopic post-surgical recurrence in asymptomatic Crohn's disease: a comparison with ultrasound. Eur Rev Med Pharacol Sci. 2006;10:17-22.

56. Yamamoto T, Shiraki M, Bamba T, et al. Faecal calprotectin and lactoferrin as markers for monitoring disease activity and predicting clinical recurrence in patients with Crohn's disease after ileocolonic resection: A prospective pilot study. United European Gastroenterol J. 2013;1:368-374. 
57. Wright EK, Kamm MA, De Cruz P, et al. Measurement of fecal calprotectin improves monitoring and detection of recurrence of Crohn's disease after surgery. Gastroenterol. 2015;148:938-947.

58. Boschetti G, Laidet M, Moussata D, et al. Levels of fecal calprotectin are associated with the severity of postoperative endoscopic recurrence in asymptomatic patients with Crohn's disease. Am J Gastroenterol. 2015;110:865-872.

59. Yamamoto T. The clinical value of faecal calprotectin and lactoferrin measurement in postoperative Crohn's disease. United European Gastroenterol. 2014;3:5-10.

60. Thomas P, Rihani H, Roseth A, et al. Assessment of ileal pouch inflammation by single-stool calprotectin assay. Dis Colon Rectum. 2000;43:214-220.

61. Johnson M, Maestranzi S, Duffy A, et al. Faecal calprotectin: a noninvasive diagnostic tool and marker of severity in pouchitis. Eur $J$ Gastroenterol Hepatol. 2008;20:174-179.

62. Pakarinen M, Koivusalo A, Natursen J, et al. Fecal calprotectin mirrors inflammation of the distal ileum and bowel function after restorative proctocolectomy for paediatric-onset ulcerative colitis. Inflamm Bowel Dis. 2010;16:482-486.

63. Tibble J, Sigthorsson G, Foster R, Sherwood R, Fagerhol M, Bjarnason I. Faecal calprotectin and faecal occult blood tests in the diagnosis of colorectal carcinoma and adenoma. Gut. 2001;49:402-408.

64. Hoff G, Grotmel T, Thiis-Evensen E, Bretthauer M, Gondal G, Vatn MH. Testing for faecal calprotectin (PhiCal) in the Norwegian Colorectal Cancer Prevention trial on flexible sigmoidoscopy screening: comparison with an immunochemical test for occult blood (FlexSure OBT). Gut. 2004;53:1329-1333.

65. Meucci G, D'Incà R, Maieron R, et al. Diagnostic value of faecal calprotectin in unselected outpatients referred for colonoscopy: A multicentre prospective study. Dig Liver Dis. 2010;42:191-195.

66. Tibble JA, Sigthorsson G, Foster R, et al. High prevalence of NSAID enteropathy as shown by a simple faecal test. Gut. 1999;45:362-366.
67. Maiden L. Capsule endoscopic diagnosis of nonsteroidal antiinflammatory drug-induced enteropathy. J Gastroenterol. 2009;44 (Suppl 19):64-71.

68. Shastri YM, Bergis D, Povse N, et al. Prospective multicentre study evaluating fecal calprotectin in adult acute bacterial diarrhoea. Sm J Med. 2008;121:1099-1106.

69. Nielsen HL, Engberg J, Ejlertsen T, Nielsen H. Evaluation of fecal calprotectin in Campylobacter concisus and Camplylobacter jejuni/ coli gastroenteritis. Scand J Gastroenterol. 2013;48:633-635.

70. Sykora J, Siala K, Humi M, Varvarovská J, Schwarz J, Pomahacová R. Evaluation of faecal calprotectin as a valuable non-invasive marker in distinguishing gut pathogens in young children with acute gastroenteritis. Acta Paediatr. 2010;99:1389-1395.

71. Chen CC, Huang JL, Chang CJ, Kong MS. Fecal calprotectin as a correlative marker in clinical severity of infectious diarrhoea and usefulness in evaluating bacterial or viral pathogens in children. $J$ Pediatr Gastroenterol Nutr. 2012;55:541-547.

72. Tursi A, Brandimarte G, Elisei W, Giorgetti GM, Inchingolo CD, Aiello F. Faecal calprotectin in colonic diverticulitis: a case-control study. Int J Colorectal Dis. 2009;24:49-55.

73. Tursi A. Biomarkers in diverticular disease of the colon. Dig Dis. 2012;30:12-18.

74. Sherwood RA. Faecal markers of gastrointestinal inflammation. J Clin Path. 2012;65:981-985.

75. Lehmann FS, Burri E, Beglinger C. The role and utility of faecal markers in inflammatory bowel disease. Ther Adv Gastroenterol. $2015 ; 8: 23-36$

76. Yang Z, Clark N, Park KT. Effectiveness and cost-effectiveness of measurement of fecal calprotectin in the diagnosis of inflammatory bowel disease. Clin Gastroenterol Hepatol. 2014;12:253-262.
Clinical and Experimental Gastroenterology

\section{Publish your work in this journal}

Clinical and Experimental Gastroenterology is an international, peerreviewed, open access journal, publishing all aspects of gastroenterology in the clinic and laboratory, including: Pathology, pathophysiology of gastrointestinal disease; Investigation and treatment of gastointestinal disease; Pharmacology of drugs used in the alimentary tract;

\section{Dovepress}

Immunology/genetics/genomics related to gastrointestinal disease. This journal is indexed on CAS. The manuscript management system is completely online and includes a very quick and fair peer-review system. Visit http://www.dovepress.com/testimonials.php to read real quotes from published authors. 